\title{
Effects of insecticides on lesser mealworm (Alphitobius diaperinus) - bioaccumulation, mortality, and growth
}

\author{
N. Meijer ${ }^{1 *}$ iD, T. de Rijk¹, J.J.A. van Loon ${ }^{2}$, M.W. Bosch ${ }^{3}$ and H.J. van der Fels-Klerx ${ }^{1}$ \\ ${ }^{1}$ Wageningen Food Safety Research, Akkermaalsbos 2, 6708 WB, Wageningen, the Netherlands; ${ }^{2}$ Wageningen University, \\ Department of Plant Sciences, Laboratory of Entomology, Droevendaalsesteeg 1, 6708 PB Wageningen, the Netherlands; \\ ${ }^{3}$ Protifarm NV, Harderwijkerweg 141B, 3852 AB Ermelo, the Netherlands; nathan.meijer@wur.nl
}

Received: 6 September 2022 / Accepted: 12 November 2021

(c) 2021 Wageningen Academic Publishers

OPEN ACCESS CC) (1) RESEARCH ARTICLE

\begin{abstract}
The lesser mealworm (LMW, Alphitobius diaperinus (Panzer); Coleoptera: Tenebrionidae) is increasingly used as a novel food and feed source. The feed materials on which this insect species is reared may contain residues of insecticides from agricultural use. The objective of this study was to determine whether selected insecticides, spiked to the substrate, could bioaccumulate in LMW and/or affect growth or survival. Insecticides were selected and spiked at concentrations equal to the legal limit in EU. Tested substances were chlorpyrifos, propoxur, imidacloprid, spinosad, tebufenozide, fipronil, pirimiphos methyl, cypermethrin, and the synergist piperonyl butoxide (PBO). Cypermethrin and PBO were also tested together. Insecticide concentrations in the spiked substrate and larvae were determined by LC-MS/MS. Concentrations in the larvae were largely below the limit of quantification, meaning that bioaccumulation did not occur. Significant reductions in total yield were observed for spinosad (present in the substrate at $1.6 \mathrm{mg} / \mathrm{kg}$ ) and imidacloprid $(0.12 \mathrm{mg} / \mathrm{kg})$. Spinosad is one of few insecticides that is permitted to be used in organic agriculture, which raises questions over the safety of organic produce for insect rearing. More research on the safety of organic produce treated with this insecticide for rearing of LMW is therefore highly recommended. The reported harmful effects of insecticide residues on LMW need to be accounted for by lowering the current legal limits or reconsidering approval for use. More research is advised particularly on sublethal effects of insecticide residues on LMW on adult beetles.
\end{abstract}

Keywords: safety, pesticides, residues, mode of action, Coleoptera

\section{Introduction}

Certain insect species are increasingly seen as an alternative protein source for food and feed (Van Huis et al., 2013). One such species is the lesser mealworm (LMW, Alphitobius diaperinus (Panzer); Coleoptera: Tenebrionidae) (Adámková et al., 2016; Janssen et al., 2017; Oonincx et $a l ., 2020)$. This new type of livestock may be exposed to pesticide residues that can remain in the feed materials on which the insects are reared. Maximum residue limits (MRLs) for pesticides have been set in EU legislation at the lowest level achievable, 'based on good agricultural practice and the lowest consumer exposure necessary to protect vulnerable consumers' (Regulation (EC) No $396 / 2009$, Article 3.2(d)). In principle, MRLs laid down in
Regulation (EC) No 396/2005 are applicable to both food and feed products (Article 18(1)). However, the effects of pesticide residues in plant-based materials in the context of rearing insects for food and feed have thus far not been accounted for.

Potential problems for insect farmers are twofold: firstly, the presence of insecticide residues in feed materials may reduce survival or induce a variety of adverse sublethal effects on growth, fecundity, and/or development (Desneux et al., 2007; Guedes et al., 2011). In a previous study, negative effects on the growth and survival of black soldier fly larvae (BSFL, Hermetia illucens (L.); Diptera: Stratiomyidae) were reported (Meijer et al., 2021). Since BSFL are phylogenetically unrelated to LMW, results may 
not be directly comparable and the need for more research on the effects of pesticide residues on more species of insects reared for food and feed is evident. Secondly, certain substances may bio-accumulate in the insects. Elevated concentrations could raise concerns on the safety of such products for consumers (Finke et al., 2015).

The focus of literature on the susceptibility of LMW to pesticides has thus far largely been on controlling this species as a pest in poultry farms. These experiments centred primarily on residual and topical contact toxicity (i.e. treated surfaces) (Despins et al., 1991; Hamm et al., 2006; Hickmann et al., 2018; Kaufman et al., 2008; Lambkin and Rice, 2007; Lyons et al., 2017; Oliveira et al., 2016; Salin et al., 2003; Steelman, 2008; Tomberlin et al., 2014; Vaughan and Turner Jr, 1984; Yeasmin et al., 2014, 2015; Zafeiriadis et al., 2021). The predictive value of such data for field conditions is limited (Stark et al., 1995); which may also be the case for commercial rearing conditions - where exposure would be via consumption of homogenised feed. The number of studies that investigated the susceptibility of LMW to pesticides via the substrate appears to be limited, focusing on the category of insect growth regulators (Singh and Johnson, 2013; Zorzetti et al., 2015). Looking at taxonomically similar species, we identified one study that evaluated the susceptibility of adults, small and large larvae of yellow mealworm (YMW, Tenebrio molitor (L.): Coleoptera: Tenebrionidae) to four types of insecticides (pirimiphos-methyl, deltamethrin, spinosad and silicoSec) via contaminated feed, in the context of these substances being used as grain protectants (Kavallieratos et al., 2019). Authors reported YMW adults to be more susceptible to these insecticides than the larvae. In particular, pirimiphosmethyl caused high mortality in small larvae and differences in effects were found depending on the type of grain the insects were reared on (barley, wheat, or maize).
The specific aims of this study were to determine the susceptibility of LMW to insecticide residues in the feed and to assess the potential bioaccumulation of these substances.

\section{Methods and materials}

In this study, LMW were exposed to a variety of insecticidal substances that had been mixed with the substrate on which the insects were reared. Eight different insecticides with different modes of action, and one synergist, were selected. Intended concentrations of these substances in the feed substrate were equal to the applicable MRL for maize. The LMW were reared on this spiked substrate for 14 days, since the younger larval instars are likely most susceptible to the effects of the insecticides. After the experiment, the larvae were killed by freezing. Susceptibility of the LMW to these substances was determined by comparing total yield, individual larval weight, and number of surviving larvae between treatments. Concentrations of insecticides in the larvae were analysed by LC-MS/MS. Bioaccumulation of tested substances was calculated, based on analysed concentrations in the larvae and substrate.

\section{Selection of insecticidal substances}

The insecticides investigated in this study - including the chemical class and mode of action (as defined by IRAC (Sparks et al., 2020) and applicable MRL for maize - are shown in Table 1. The selection of substances was based on our previous study on BSFL (Meijer et al., 2021), covering the following substances: chlorpyrifos, propoxur, imidacloprid, spinosad, tebufenozide, cypermethrin, piperonyl butoxide (PBO). Imidacloprid as an active substance in plant protection products was still permitted to be used as a seed coating for crops grown in permanent greenhouses at the time that the experiments were conducted (Regulation (EU) No 2018/783). Although the EU-wide approval has

Table 1. Selected insecticides including class, mode of action, and maximum residue level (MRL) in the EU for maize (Regulation (EC) No 396/2005).

\begin{tabular}{llll} 
Substance name & Class & Mode of Action & MRL (mg/kg) \\
Chlorpyrifos & Organophosphates & Acetylcholinesterase (AChE) inhibitors & 0.05 \\
Propoxur & Carbamates & AChE inhibitors & $0.05^{*}$ \\
Imidacloprid & Neonicotinoids & Nicotinic acetylcholine receptor (NAchR) competitive modulators & 0.1 \\
Spinosad & Spinosyns & NAchR allosteric modulators - site I & 2.0 \\
Tebufenozide & Insect growth regulators & Ecdysone receptor agonists & $0.05^{*}$ \\
Fipronil & Phenylpyrazoles (fiproles) & GABA-gated chloride channel blockers & $0.005^{*}$ \\
Pirimiphos methyl & Organophosphates & AChE inhibitors & 0.5 \\
Cypermethrin & Pyrethroids & Sodium channel modulators & 0.3 \\
Piperonyl butoxide & Synergist & Synergist & no MRL \\
\hline${ }^{1}$ An asterisk indicates lower limit of analytical determination. & &
\end{tabular}


expired since then (Regulation (EU) 2020/1643), individual EU member states have authorised imidacloprid for various uses, using emergency derogation powers (Article 53 of Regulation (EC) No 1107/2009).

In addition to the selection of insecticides in Meijer et al. (2021), the phenylpyrazole fipronil and organophosphate pirimiphos-methyl were also tested in this study. Pirimiphos-methyl was added because of the high mortality found in the taxonomically related YMW (Kavallieratos et al., 2019). Fipronil was included to test an additional mode of action: GABA-gated chloride channel blockers (SimonDelso et al., 2015; Sparks et al., 2020).

The effects of selected insecticides on LMW were tested in two sequential experiments. In the first experiment ('Exp. $\left.1^{\prime}\right)$, the substances chlorpyrifos, propoxur, imidacloprid, spinosad, tebufenozide, fipronil, and pirimiphos methyl were tested. This experiment was performed with each treatment in triplicate. In the second experiment ('Exp. 2'), the effects of cypermethrin and PBO were tested with 5 replicates per treatment. Exp. 2 was held at a smaller scale than Exp. 1. The larger number of replicates in Exp. $2(n=5)$ were used to be able to determine whether the presumed synergistic effects of $\mathrm{PBO}$ were statistically significant, by comparing the results of the treatment containing both cypermethrin and $\mathrm{PBO}$ to the treatment containing only cypermethrin. The other substances tested in Exp. 1 were compared against the (pooled, $n=9$ ) controls, for which $\mathrm{n}=3$ per treatment were considered to suffice.

\section{Feed preparation}

A test feed ('substrate') was composed for both experiments, consisting of a dry mix of primarily organic wheat products, a vegetable protein source, and a pre-mix (Research
Diet Services, Wijk bij Duurstede, the Netherlands). This substrate was provided by Protifarm B.V. (Ermelo, the Netherlands), where the experiments took place. The substrate was prepared for the experiments at the Wageningen Food Safety Research (WFSR) laboratory by spiking with insecticides dissolved in either acetonitril $(\mathrm{ACN})$ or methanol $(\mathrm{MeOH})$. The intended concentration of each insecticide was equal to the selected MRL (Table 1). Since $P B O$ is not a plant protection product, it does not have an MRL. Therefore, it was used at a ratio of 1:20 (cypermethrin:PBO), which is a ratio used in commercial formulations for application of this pyrethroid / synergist combination in the field (Osimitz, 2010), similar to Meijer et al. (2021). The list of treatments, spiked concentrations, solvent, purity (\%), and suppliers is provided in Table 2.

Based on practical experiences, $0.275 \mathrm{~kg}$ of feed would be required per replicate for Exp. 1 (chlorpyrifos, propoxur, imidacloprid, spinosad, tebufenozide, fipronil, and pirimiphos methyl). Therefore, per treatment at least 0.825 $\mathrm{kg}$ of feed $(3 \times 0.275)$ was required to be spiked. In order to mix the insecticides homogenously with the provided dry feed, tap water was added before the spiking and subsequent mixing. Per treatment, $1 \mathrm{~kg}$ of feed was mixed with 31 water, using a UM 12 table-top mixer (Stephan Machinery GmbH, Hameln, Germany) at 1,500 rpm for 2 min. The intended concentration, and therefore the total volume of insecticides and respective solvents, differed per treatment. In order for the volume of solvents to be equal in all treatments, additional $\mathrm{MeOH}$ or $\mathrm{ACN}$ was added: bringing the total volume to $1.0 \mathrm{ml}(\mathrm{MeOH})$ and $3.5 \mathrm{ml}$ (ACN) per treatment, respectively. The same volumes were also added to the two respective solvent controls. The resulting slurry was moved into 3 plastic zipper bags each per treatment, which were put into crates, and were then frozen at $-18{ }^{\circ} \mathrm{C}$. The frozen spiked substrate was freeze-

Table 2. Insecticides tested, their intended spiked concentration in the feed, solvent, purity, and suppliers.

\begin{tabular}{|c|c|c|c|c|}
\hline Substance & Spiked concentration (mg/kg) & Solvent & Purity (\%) & Supplier \\
\hline Chlorpyrifos & 0.05 & methanol & 99.3 & Sigma-Aldrich ${ }^{1}$ \\
\hline Propoxur & 0.05 & methanol & 99.9 & $\mathrm{HPC}^{2}$ \\
\hline Imidacloprid & 0.1 & methanol & 98.7 & $\mathrm{HPC}^{2}$ \\
\hline Spinosad & 2.0 & methanol & 96.6 & $\mathrm{HPC}^{2}$ \\
\hline Tebufenozide & 0.05 & acetonitrile & 99.9 & Sigma-Aldrich ${ }^{1}$ \\
\hline Fipronil & 0.005 & methanol & 97.3 & $\mathrm{HPC}^{2}$ \\
\hline Pirimiphos-methyl & 0.5 & acetonitrile & 98.5 & Sigma-Aldrich ${ }^{1}$ \\
\hline Cypermethrin & 0.3 & acetonitrile & 99.7 & $\mathrm{HPC}^{2}$ \\
\hline Piperonyl butoxide (PBO) & 6.0 & acetonitrile & 92.5 & Dr. Ehrenstorfer ${ }^{3}$ \\
\hline Cypermethrin + PBO & $0.3+6.0$ & acetonitrile & $99.7+92.5$ & $\mathrm{HPC}^{2}+\mathrm{Dr}$. Ehrenstorfer ${ }^{3}$ \\
\hline
\end{tabular}


dried in batches in a pilot scale tray freeze dryer (Scala Scientific, Ede, the Netherlands) at Unifarm (Wageningen University \& Research, Wageningen, the Netherlands) until the shelf temperature was approximately equal to the product temperature.

In Exp. 2, the following four treatments were tested: only cypermethrin, only $\mathrm{PBO}$, both cypermethrin and $\mathrm{PBO}$, and a blank control. It was decided to only include a blank control (and no solvent control) in Exp. 2 because the differences of solvent controls with the blank control in Exp. 1 showed not to be significant $(P>0.05)$. The feed preparation procedure in Exp. 2 was the same as in Exp. 1, except that: (1) $75 \mathrm{~g}$ of feed was used per replicate; and (2) the spiked feed was freeze-dried using a Millrock REVO Series Freeze-Dryer Model LD 85 (Millrock Technology, Kingston, NY, USA) at WFSR (Wageningen, The Netherlands).

\section{Experimental procedures}

For Exp. 1, per replicate, $1.85 \mathrm{~g}$ of first instars LMW, originating from one cohort of eggs, were weighed and put in a small container $(19 \times 11.5 \mathrm{~cm}$, Dampack International, Werkendam, the Netherlands). The containers were prepared with a small amount of dry meal and a small amount of wetted meal (water to meal ratio 2:1), to provide moisture. During the 14-day rearing period, additional wetted meal was provided daily - amounts corresponding to the growth of the larvae. Over the 14-day rearing period, $275 \mathrm{~g}$ of meal was provided in total per replicate. During feeding, for every treatment, clean gloves were used to avoid cross-contamination of the different treatments. After 14 days, the larvae were separated from the feed substrate residue by sieving. The insects were thoroughly cleaned by shaking the insects over a $1 \mathrm{~mm}$ sieve so all attached faecal and feed particles were removed from the animals. The insects were weighed to determine total yield (g) per replicate. For each replicate, a representative sample (600-800 mg), accurately weighed, was taken. The number of larvae in this sample was counted twice to calculate the average individual weight of the larvae (mg). This individual larval weight was used to calculate the total number of larvae in the replicate (based on total weight of the replicate). Finally, insects were killed by freezing at $-18^{\circ} \mathrm{C}$.

The animal procedures of Exp. 2 were identical to Exp. 1 , with the exception that $0.4 \mathrm{~g}$ of first instar LMW were reared per replicate, and a smaller container $(11.5 \times 11.5 \mathrm{~cm}$, Dampack International) was used - both to account for the reduced amount of substrate that was provided in Exp. 2 .

\section{Chemical analyses and quality control}

Analyses were performed using the method applied in Meijer et al. (2021). In brief, the active compounds were extracted from the frozen larval samples $(1.0 \pm 0.05 \mathrm{~g})$ and analysed by liquid chromatography-mass spectrometry (LC/ MS-MS). UPLC gradient conditions for all substances are listed in Table S1. MS/MS conditions of the compounds fipronil and pirimiphos-methyl, which were additional to the mentioned previous study, are presented in Table S2.

Quality control was performed by spiking blank samples with active substances in ranges corresponding to the spiked concentration (Table S3).

\section{Calculations and statistical analyses}

The bioaccumulation factor of tested substances was calculated by dividing the analysed concentration in the larvae by the concentration in the substrate, applying the procedure used in Van der Fels-Klerx et al. (2016).

Statistical analyses were performed using the software SPSS Statistics for Microsoft Windows (version 25.0.0.2, IBM Corp., Armonk, NY, USA). Non-parametric statistical tests were used because the number of replicates per treatment did not warrant tests on conformity to a distribution type. Statistically tested variables were total yield (g), individual larval weight (mg), and number of larvae (n). For Exp. 1 $(n=3)$, differences in each of these three variables between the blank and two solvent controls were analysed using Kruskal-Wallis tests $(\alpha=0.05)$. If differences were not significant for the three controls $(P>0.05)$, the controls were pooled (i.e. $n=9$ ) to compare the values of the controls with those of the spiked treatments (Kruskal-Wallis tests $(\alpha=0.05))$. For variables for which differences were significant $(P<0.05)$, results for each spiked treatment were compared to the pooled controls using a Mann-Whitney U test. Because this post-hoc test was a multiple comparison test, a lower significance level of 0.01 was used.

The same statistical tests were used for Exp. 2, but the treatments were compared against the blank control $(n=5)$ that was used in that experiment (Kruskal-Wallis, $\alpha=0.05$ ). For variables for which differences were significant $(P<0.05)$, results for each spiked treatment were compared to the control using a Mann-Whitney U test $(\alpha=0.01)$. Finally, for each of the variables, differences between the treatment containing cypermethrin were compared to the treatment that contained both cypermethrin and PBO, using a MannWhitney $\mathrm{U}$ test $(\alpha=0.01)$. 


\section{Results}

\section{Quality control}

The results of the quality control analyses of the substrate are shown in Table S3. For all treatments, except for pirimiphos-methyl, the mean recovery was within the acceptable range of 70-120\% (EC, 2019). For pirimiphosmethyl, recovery was $65.1 \%$. Recovery correction is acceptable when the recovery is outside of the range of $80-120 \%$ (but within $30-140 \%$ ), and in that case the recovery should be multiplied with a factor (100\%/recovery \%) (EC, 2019). The measured concentration for pirimiphos-methyl (of $0.42 \mathrm{mg} / \mathrm{kg}$ ) was therefore corrected to $0.64 \mathrm{mg} / \mathrm{kg}$.

The results of the quality control analyses of the larvae are shown in Table S4. The average recovery of fipronil in the larval samples was insufficient. Hence, we do not present concentrations in the larvae and the bioaccumulation factor of fipronil.

\section{Larval survival and growth}

Results for the variables total yield, individual larval weight, and number of larvae (combined for both experiments) are shown in Table S5. For number of larvae, yield and individual larval weight; differences were not significant between the blank and two solvent controls $(P>0.05)$ in Exp. 1. Therefore, the three controls $(n=9)$ were pooled for further statistical analysis.

In Exp. 1, differences in total yield $(P=0.05)$ and individual larval weight $(P=0.046)$ were significant, but the number of larvae was not affected $(P=0.267)$. The post-hoc tests showed a significant reduction in total yield by imidacloprid $(P=0.009)$ and spinosad $(P=0.009)$. The post-hoc tests for individual larval weight showed no significant differences between each of the spiked treatments and the pooled controls $(P>0.01)$.

In Exp. 2, differences were significant for total yield ( $P=0.03$ ) and individual larval weight $(P=0.024)$ but, similar to Exp. 1 , the number of larvae did not differ $(P=0.268)$. The post-hoc tests for total yield and individual larval weight showed no significant differences between any of the treatments and the control $(P>0.01)$. However, differences between the treatment containing only cypermethrin and the treatment containing both cypermethrin and PBO were significant, both for total yield $(P=0.008)$ and individual larval weight $(P=0.008)$.

Results for total yield and individual larval weight are shown in Figure 1 and Figure 2, respectively, combined for both experiments. The values in the boxplots for each of the treatments are expressed as a percentage of the mean control values in the respective experiment. For Exp. 1 (chlorpyrifos, propoxur, imidacloprid, spinosad, tebufenozide, fipronil, and pirimiphos-methyl), this was the mean of the pooled controls, whereas this was the blank control for Exp. 2 (cypermethrin and PBO).

\section{Concentrations and bioaccumulation}

Table 3 presents the concentrations of the substances in the larvae. For almost all tested substances, concentrations in the larvae were below their respective limit of quantification

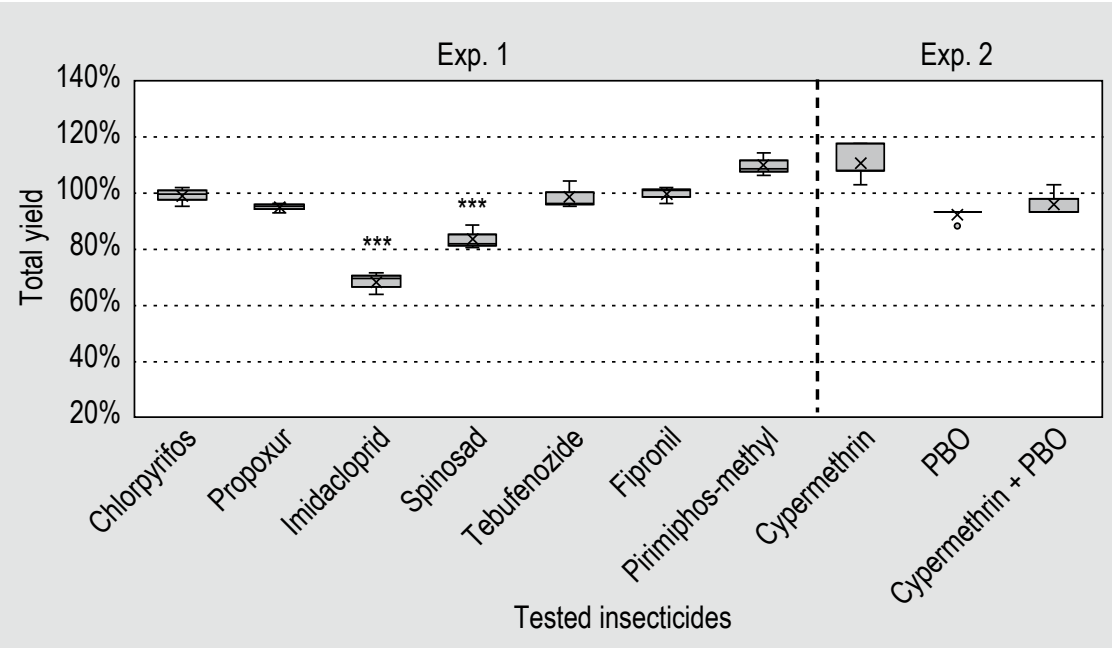

Figure 1. Percentage of total larval yield for each of the treatments (box plots). For Exp. 1 (chlorpyrifos, propoxur, imidacloprid, spinosad, tebufenozide, fipronil, and pirimiphos-methyl), this is expressed as the percentage of the treatment $(n=3)$ relative to the mean of the pooled values for the blank and two solvent controls ( $n=9$ ). For Exp. 2 (cypermethrin and piperonyl butoxide (PBO) (and the treatment combining both)), the mean of the blank control (Exp. 2; $n=5$ ) was used as the reference in the calculation of the percentage. Significance of differences between a treatment and the respective control(s) $(P<0.01)$ is denoted by ${ }^{* * *}$. 


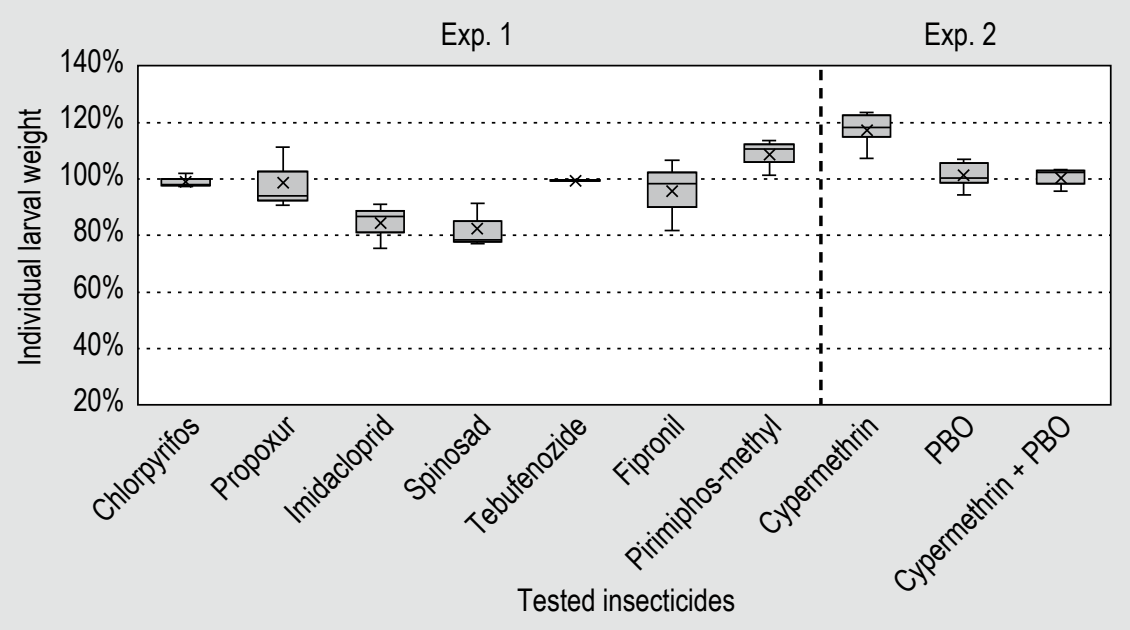

Figure 2. Percentage of individual larval weight for each of the treatments (box plots). For Exp. 1 (chlorpyrifos, propoxur, imidacloprid, spinosad, tebufenozide, fipronil, and pirimiphos-methyl), this is expressed as the percentage of the treatment $(\mathrm{n}=3)$ relative to the mean of the pooled values for the blank and two solvent controls $(n=9)$. For Exp. 2 (cypermethrin and piperonyl butoxide (PBO) (and the treatment combining both)), the mean of the blank control (Exp. 2; $n=5$ ) was used as the reference in the calculation of the percentage.

Table 3. Concentrations ( $\mathrm{mg} / \mathrm{kg}$ ) of tested insecticides in spiked feed (pre-experiment) and larvae (post-experiment) as quantified by LC-MS.

\begin{tabular}{lll} 
Substance & Concentration feed $(\mathbf{m g} / \mathbf{k g})$ & Concentration larvae $(\mathbf{m g} / \mathbf{k g})$ \\
Chlorpyrifos & 0.04 & $<\mathrm{LOQ}(0.005)$ \\
Propoxur & 0.04 & $<\mathrm{LOQ}(0.001)$ \\
Imidacloprid & 0.12 & $<\mathrm{LOQ}(0.001)$ \\
Spinosad & 1.58 & 0.001 \\
Tebufenozide & 0.05 & $<\mathrm{LOQ}(0.005)$ \\
Fipronil & 0.01 & $\mathrm{n} / \mathrm{a}{ }^{1}$ \\
Pirimiphos methyl & $0.64^{2}$ & $<\mathrm{LOQ}(0.001)$ \\
Cypermethrin & 0.38 & $<\mathrm{LOQ}(0.025)$ \\
Piperonyl butoxide (PBO) & $<\mathrm{LOQ}(0.025)$ \\
Cypermethrin + PBO & 5.1 & $<\mathrm{LOQ}(0.025)$ \\
& $0.28+5.1$ & \\
\hline & & \\
& &
\end{tabular}

$(<\mathrm{LOQ})$, except for spinosad, which had a very low concentration in the larvae. Given the insecticide being absent or very low in the larvae, accurate bio-accumulation factors could, not be calculated.

\section{Discussion}

Five out of eight insecticides tested in this study (chlorpyrifos, propoxur, tebufenozide, fipronil, and pirimiphos-methyl) did not significantly affect total yield, individual larval weight, and number of surviving larvae of LMW. However, the absence of significant effects in this experiment does not exclude the possibility of adverse effects in life-stages other than the larvae. Differences in susceptibility to pesticides - depending on the life stage of an insect (adult/larvae) (Hickmann et al., 2018; Zafeiriadis et al., 2021) and manner of application (topical / residual) (Steelman, 2008) - have been observed for LMW and other Tenebrionidae (Kavallieratos et al., 2019). Furthermore, it is also possible that exposure to (sub-) lethal concentrations of pesticides in the diet manifest as adverse effects in a later life-stage; e.g. by affecting pupation, adult emergence, or fecundity (De França et al., 2017; Desneux et al., 2007; Haynes, 1988). More research is therefore required to 
determine the susceptibility of other LMW life-stages when exposed to insecticide residues in the substrate.

The advice to study the effects of insecticides on other LMW life stages is especially pertinent for insect growth regulators, such as tebufenozide tested in this study. Tebufenozide is a synthetic ecdysone agonist that interferes with the moulting process, thereby halting development (Barnett and Brundage, 2010). Tebufenozide is most effective against Lepidoptera, but less so against Coleoptera (Nakagawa, 2005). Tebufenozide was initially selected due to the presence of residues found in feed materials, reported in RASFF (Meijer et al., 2021).

A significant reduction in total yield was observed in the two treatments containing imidacloprid $(68.4 \pm 4.0 \%$ of mean value of pooled controls) and spinosad $(83.7 \pm 4.2 \%)$. The susceptibility of LMW to both substances is well studied in terms of topical and residual toxicity (Singh and Johnson, 2015; Yeasmin et al., 2015; Zafeiriadis et al., 2021). Both compounds target the nicotinic acetylcholine receptor (Casida, 2009; Sparks et al., 2020). The highest concentration in this study $(2.0 \mathrm{mg} / \mathrm{kg})$ was used for spinosad, and this high dose is thought to play a role in the observed effects. A concentration in the range of 0.005-0.5 $\mathrm{mg} / \mathrm{kg}$, like the other tested substances, would be expected to cause substantially weaker effects. Notably, spinosad is one of the few insecticides that is permitted to be used as a plant protection product in organic farming in the EU (Regulation (EC) No 889/2008, Annex II). This raises questions over the presumed safety of organic produce for LMW rearing if spinosad residues persist in the feed on which the insects are reared. Imidacloprid caused a more severe reduction in total yield, despite the lower concentration used $(0.1 \mathrm{mg} / \mathrm{kg})$, compared to spinosad's $(2.0$ $\mathrm{mg} / \mathrm{kg}$ ). Although the EU-wide approval of imidacloprid has expired, it is still permitted to be used in several countries by derogation, and feed materials may therefore continue to contain residues of imidacloprid at low concentrations. The results of this study suggest that such concentrations can cause mortality in LMW.

Differences in total yield and individual larval weight were significant between the treatment containing only cypermethrin, and the treatment containing both cypermethrin and $\mathrm{PBO}$, but not between either of these treatments and the control. Cypermethrin appeared to cause a slight increase in total yield as compared to the control $(110.8 \pm 6.6 \%)$, while the addition of PBO caused a minor decrease $(96.1 \pm 4.4 \%)$. We could not identify any published study on hormesis in Coleoptera by cypermethrin. It is, therefore, unclear what could have caused this increase in total yield.

The concentrations of almost all tested substances - except spinosad, which was very low $(0.001 \mathrm{mg} / \mathrm{kg})$ - in the larvae were below the limit of quantification. Concerns over bioaccumulation of these substances can, therefore, be ruled out and the presence of residues of these insecticides at the concentrations in the substrate tested is not likely to pose a safety risk for the animal or human that will be consuming the final insect product.

The concentration of fipronil in the larvae is not reported because recovery of this substance in the quality control analyses was insufficient. It is unclear what may have caused this. One possible explanation could be interference in the analysis by the larval matrix.

It must be highlighted that the results of this study may be limited to the particular LMW strain that was used in these experiments. Several studies have found significant interpopulation variation in susceptibility of LMW, collected from poultry farms, to a variety of pesticides, including the substances imidacloprid, spinosad, and cypermethrin which significantly affected LMW larvae in this study (Hickmann et al., 2018; Renault and Colinet, 2021; Singh and Johnson, 2015). Resistance to the pesticides reported in the latter studies correlated to long-term exposure of populations to sub-lethal doses. The LMW strain used in this study has been reared in captivity for over 35 years. Chronic exposure to sub-lethal insecticide concentrations in that time is possible. Therefore, the particular strain that was used for these experiments may have developed resistance to the tested substances, that may not be present in other populations. It is therefore advisable to verify these results in other commercial LMW strains.

\section{Conclusion and recommendations}

The aims of this study were to determine the susceptibility of LMW to insecticide residues in the feed and to assess the potential bioaccumulation of these substances. This was done for the active substances chlorpyrifos, propoxur, imidacloprid, spinosad, tebufenozide, fipronil, pirimiphosmethyl, and cypermethrin. The latter substance was also tested in combination with the synergist PBO. We conclude that growth and survival of LMW may be negatively affected by spinosad and imidacloprid at insecticide residue concentrations that are currently allowed in animal feed in the EU. Cypermethrin exposure resulted into an increase in LMW total yield and individual larval weight, but the addition of PBO negated this effect. All concentrations of the tested substances in the larvae were $<$ LOQ and bioaccumulation must thus be assumed to be nil, given the insecticide concentrations around MRL levels in the substrate. LMW products from insects exposed to residues of tested insecticides during rearing at concentrations equal to the MRL can therefore be considered safe for consumers.

Based on our results, we strongly recommend policymakers to consider adopting insect species-specific MRLs for 
feed materials. The effects of insecticides on commercial insect rearing for food and feed should also be taken into account for the approval and legal limits of insecticides, as is already being done for beneficial insects such as pollinators. More research should be done on the safety for insects reared on organic feed treated with spinosad. Insect farmers are advised to be diligent in analysing incoming feed materials for the presence of insecticide residues, as this may significantly affect total yield. More research is needed on a variety of insecticidal substances. In addition, it is encouraged to assess potential sub-lethal effects of insecticides on other life-stages of the insects than the larval stage, in particular the adult stage essential for reproduction, and using other commercial insect strains.

\section{Supplementary material}

Supplementary material can be found online at https://doi. org/10.3920/JIFF2021.0157.

Table S1. UPLC Gradient conditions for all tested substances.

Table S2. MS/MS conditions for the substances fipronil and pirimiphos-methyl.

Table S3. Quality control results analytical procedure (substrate), for all tested substances.

Table S4. Quality control results analytical procedure (larvae), for all tested substances.

Table S5. Total yield, individual larval weight, and number of larvae per treatment.

\section{Acknowledgements}

We wish to thank the following people for their contributions to this study:

- Paul Zomer and Joost Memelink (WFSR) for performing the LC-MS/MS analyses

- Jacqualine Derksen (WFSR) for spiking the substrates

- Niek Steeghs (Protifarm) for providing resources and technical input on LMW rearing

- Elise Hoek (WFSR) for advising on the research design and statistical analyses

- Bert Brouwer (WFSR) for freeze-drying the substrate in the experiment with cypermethrin and PBO

- Marcel Visser and Patrick Hendrickx (Unifarm) for freeze-drying the substrate in the experiment with chlorpyrifos, propoxur, imidacloprid, spinosad, tebufenozide, fipronil, and pirimiphos-methyl

\section{Conflict of interest}

This project was funded by the Netherlands Ministry of Economic Affairs (further: The Ministry) through a Public-Private Partnership project ('Controlling the safety of insects for food and feed') of the Topsector AgriFood (TKI-AF-15220). The Ministry had no role in study design, data collection and analysis, decision to publish, or preparation of the manuscript. The commercial entities within the project consortium were: Proti-Farm R\&D, BV; Protix Biosystems; Bestico B.V., and ForFarmers. With the exception of Proti-Farm R\&D, BV, the commercial entities in the consortium had no role in study design, data collection and analysis, decision to publish, or preparation of the manuscript. Proti-Farm R\&D, BV is a subsidiary of SAS Ÿnsect. Proti-Farm R\&D, BV produces lesser mealworm for food. Proti-Farm R\&D, BV provided in-kind contribution to the project primarily in the form of labour costs for author M.W.B., who assisted in the execution of experiments, which were performed at the premises of that company under supervision of the corresponding author, and for reviewing and editing the manuscript prior to submission. Proti-Farm R\&D, BV otherwise had no role in the study design, data collection and analysis, decision to publish, or preparation of the manuscript.

\section{References}

Adámková, A., Kouřimská, L., Borkovcová, M., Kulma, M. and Mlček, J., 2016. Nutritional values of edible Coleoptera (Tenebrio molitor, Zophobas morio and Alphitobius diaperinus) reared in the Czech Republic. Potravinarstvo Slovak Journal of Food Sciences 10(1): 663-671. https://doi.org/10.5219/609

Barnett, J.B. and Brundage, K.M., 2010. Immunotoxicology of pesticides and chemotherapies. In: McQueen, C.A. (ed.) Comprehensive toxicology, $2^{\text {nd }}$ edition. Elsevier, Oxford, UK, pp. 467-487. https:// doi.org/10.1016/B978-0-08-046884-6.00627-8

Casida, J.E., 2009. Pest toxicology: the primary mechanisms of pesticide action. Chemical Research in Toxicology 22: 609-619.

De França, S.M., Breda, M.O., Barbosa, D.R., Araujo, A.M. and Guedes, C.A., 2017. The sublethal effects of insecticides in insects. In: Biological control of pest and vector insects. IntechOpen, London, UK, pp. 23-39.

Desneux, N., Decourtye, A. and Delpuech, J.-M., 2007. The sublethal effects of pesticides on beneficial arthropods. Annual Review of Entomology 52: 81-106.

Despins, J.L., Turner Jr, E.C. and Pfeiffer, D.G., 1991. Evaluation of methods to protect poultry house insulation from infestations by lesser mealworm (Coleoptera: Tenebrionidae). Journal of Agricultural Entomology 8: 209-217.

European Commission (EC), 2019. SANTE/12682/2019 - analytical quality control and method validation procedures for pesticide residues analysis in food and feed. EC. Brussels, Belgium. 
Finke, M.D., Rojo, S., Roos, N., Van Huis, A. and Yen, A., 2015. Scientific opinion on a risk profile related to production and consumption of insects as food and feed. EFSA Journal 13: 4257. https://doi. org/10.2903/j.efsa.2015.4257

Guedes, R.N.C., Guedes, N.M.P. and Rosi-Denadai, C.A., 2011. Sublethal effects of insecticides on stored-product insects: current knowledge and future needs. Stewart Postharvest Review 7: 1-5.

Hamm, R.L., Kaufman, P.E., Reasor, C.A., Rutz, D.A. and Scott, J.G., 2006. Resistance to cyfluthrin and tetrachlorvinphos in the lesser mealworm, Alphitobius diaperinus, collected from the eastern United States. Pest Management Science 62: 673-677.

Haynes, K.F., 1988. Sublethal effects of neurotoxic insecticides on insect behavior. Annual Review of Entomology 33: 149-168.

Hickmann, F., De Morais, A.F., Bronzatto, E.S., Giacomelli, T., Guedes, J.V. and Bernardi, O., 2018. Susceptibility of the lesser mealworm, Alphitobius diaperinus (Coleoptera: Tenebrionidae), from broiler farms of Southern Brazil to insecticides. Journal of Economic Entomology 111: 980-985.

Janssen, R.H., Vincken, J.-P., Van den Broek, L.A., Fogliano, V. and Lakemond, C.M., 2017. Nitrogen-to-protein conversion factors for three edible insects: Tenebrio molitor, Alphitobius diaperinus, and Hermetia illucens. Journal of Agricultural and Food Chemistry 65: 2275-2278.

Kaufman, P.E., Strong, C. and Rutz, D.A., 2008. Susceptibility of lesser mealworm (Coleoptera: Tenebrionidae) adults and larvae exposed to two commercial insecticides on unpainted plywood panels. Pest Management Science 64: 108-111.

Kavallieratos, N.G., Michail, E.J., Boukouvala, M.C., Nika, E.P. and Skourti, A., 2019. Efficacy of pirimiphos-methyl, deltamethrin, spinosad and silicoSec against adults and larvae of Tenebrio molitor L. on wheat, barley and maize. Journal of Stored Products Research 83: 161-167.

Lambkin, T.A. and Rice, S.J., 2007. Baseline responses of Alphitobius diaperinus (Coleoptera: Tenebrionidae) to spinosad, and susceptibility of broiler populations in eastern and southern Australia. Journal of Economic Entomology 100: 1423-1427.

Lyons, B.N., Crippen, T.L., Zheng, L., Teel, P.D., Swiger, S.L. and Tomberlin, J.K., 2017. Susceptibility of Alphitobius diaperinus in Texas to permethrin-and $\beta$-cyfluthrin-treated surfaces. Pest Management Science 73: 562-567.

Meijer, N., De Rijk, T., Van Loon, J.J.A., Zoet, L. and Van der FelsKlerx, H.J., 2021. Effects of insecticides on mortality, growth and bioaccumulation in black soldier fly (Hermetia illucens) larvae. PLoS ONE 16: e0249362.

Nakagawa, Y., 2005. Nonsteroidal ecdysone agonists. Vitamins \& Hormones 73: 131-173.

Oliveira, D., Cardoso, R., Mamprim, A. and Angeli, L., 2016. Laboratory and field evaluation of a cypermethrin-based insecticide for the control of Alphitobius diaperinus Panzer (Coleoptera: Tenebrionidae) and its in-vitro effects on Beauveria bassiana Bals. Vuill. (Hypocreales: Cordycipitaceae). Brazilian Journal of Poultry Science 18: 371-380.

Oonincx, D.G.A.B., Laurent, S., Veenenbos, M.E. and Van Loon, J.J.A., 2020. Dietary enrichment of edible insects with omega 3 fatty acids. Insect Science 27: 500-509.
Osimitz, T.G., 2010. The safety assessment of piperonyl butoxide. In: Krieger, R. (ed.) Hayes' handbook of pesticide toxicology. Elsevier, Amsterdam, the Netherlands, pp. 2127-2151.

Renault, D. and Colinet, H., 2021. Differences in the susceptibility to commercial insecticides among populations of the lesser mealworm Alphitobius diaperinus collected from poultry houses in France. Insects 12: 309.

Salin, C., Delettre, Y. and Vernon, P., 2003. Controlling the mealworm Alphitobius diaperinus (Coleoptera: Tenebrionidae) in broiler and turkey houses: field trials with a combined insecticide treatment: insect growth regulator and pyrethroid. Journal of Economic Entomology 96: 126-130.

Simon-Delso, N., Amaral-Rogers, V., Belzunces, L.P., Bonmatin, J.M., Chagnon, M., Downs, C., Furlan, L., Gibbons, D.W., Giorio, C. and Girolami, V., 2015. Systemic insecticides (neonicotinoids and fipronil): trends, uses, mode of action and metabolites. Environmental Science and Pollution Research 22: 5-34.

Singh, N. and Johnson, D., 2013. Baseline responses of Alphitobius diaperinus (Coleoptera: Tenebrionidae) to insect growth regulators. Journal of Agricultural and Urban Entomology 29: 35-54.

Singh, N. and Johnson, D., 2015. Baseline susceptibility and crossresistance in adult and larval Alphitobius diaperinus (Coleoptera: Tenebrionidae) collected from poultry farms in Arkansas. Journal of Economic Entomology 108: 1994-1999.

Sparks, T.C., Crossthwaite, A.J., Nauen, R., Banba, S., Cordova, D., Earley, F., Ebbinghaus-Kintscher, U., Fujioka, S., Hirao, A. and Karmon, D., 2020. Insecticides, biologics and nematicides: updates to IRAC's mode of action classification - a tool for resistance management. Pesticide Biochemistry and Physiology 167: 104587.

Stark, J.D., Jepson, P.C. and Mayer, D.F., 1995. Limitations to use of topical toxicity data for predictions of pesticide side effects in the field. Journal of Economic Entomology 88: 1081-1088.

Steelman, C.D., 2008. Comparative susceptibility of adult and larval lesser mealworms, Alphitobius diaperinus (Panzer) (Coleoptera: Tenebrionidae), collected from broiler houses in Arkansas to selected insecticides. Journal of Agricultural and Urban Entomology 25: 111-125.

Tomberlin, J.K., Richman, D. and Myers, H.M., 2014. Susceptibility of Alphitobius diaperinus (Coleoptera: Tenebrionidae) from broiler facilities in Texas to four insecticides. Journal of Economic Entomology 101: 480-483.

Van der Fels-Klerx, H.J., Camenzuli, L., Van der Lee, M.K. and Oonincx, D.G.A.B., 2016. Uptake of cadmium, lead and arsenic by Tenebrio molitor and Hermetia illucens from contaminated substrates. PLoS ONE 11: e0166186.

Van Huis, A., Van Itterbeeck, J., Klunder, H., Mertens, E., Halloran, A., Muir, G. and Vantomme, P., 2013. Edible insects: future prospects for food and feed security. Food and Agriculture Organization of the United Nations, Rome, Italy.

Vaughan, J. and Turner Jr, E., 1984. Residual and topical toxicity of various insecticides to the lesser mealworm (Coleoptera: Tenebrionidae). Journal of Economic Entomology 77: 216-220. 
Yeasmin, A.M., Waliullah, T.M., Islam, N. and Ashraful, M., 2015. Cotoxicity study of imidacloprid and a synergist PBO on Alphitobius diaperinus (Coleoptera: Tenebrionidae) for biological safety of stored grains and cereals. World Journal of Pharmaceutical Research 4: 254-269.

Yeasmin, A.M., Waliullah, T.M. and Rahman, A.S., 2014. Synergistic effects of chlorpyrifos with piperonyl butoxide (pbo) against the lesser mealworm, Alphitobius diaperinus (Panzer) (Coleoptera: Tenebrionidae). Asian Pacific Journal of Reproduction 3: 305-310.
Zafeiriadis, S., Sakka, M.K. and Athanassiou, C.G., 2021. Efficacy of contact insecticides for the control of the lesser mealworm, Alphitobius diaperinus (Panzer) (Coleoptera: Tenebrionidae). Journal of Stored Products Research 92: 101817.

Zorzetti, J., Constanski, K., Santoro, P.H., Fonseca, I.C. and Neves, P.M., 2015. Growth regulator insecticides for the control of the lesser mealworm beetle Alphitobius diaperinus (Coleoptera: Tenebrionidae). Revista Colombiana de Entomología 41: 24-32. 